\title{
Du comique dans le théâtre contemporain, numéro dirigé par Bernadette Bost et Mireille Losco-Lena
}

\section{Riccardo Benedettini}

\section{(2) OpenEdition}

\section{Journals}

\section{Edizione digitale}

URL: http://journals.openedition.org/studifrancesi/9343

DOI: 10.4000/studifrancesi.9343

ISSN: 2421-5856

\section{Editore}

Rosenberg \& Sellier

\section{Edizione cartacea}

Data di pubblicazione: 1 juin 2008

Paginazione: 225

ISSN: 0039-2944

\section{Notizia bibliografica digitale}

Riccardo Benedettini, «Du comique dans le théâtre contemporain, numéro dirigé par Bernadette Bost et Mireille Losco-Lena», Studi Francesi [Online], 154 (LII | I) | 2008, online dal 30 novembre 2015, consultato il 09 janvier 2021. URL: http://journals.openedition.org/studifrancesi/9343 ; DOI: https:// doi.org/10.4000/studifrancesi.9343

Questo documento è stato generato automaticamente il 9 janvier 2021.

\section{(c)}

Studi Francesi è distribuita con Licenza Creative Commons Attribuzione - Non commerciale - Non opere derivate 4.0 Internazionale. 


\title{
Du comique dans le théâtre contemporain, numéro dirigé par Bernadette Bost et Mireille Losco- Lena
}

\author{
Riccardo Benedettini
}

\section{NOTIZIA}

Du comique dans le théâtre contemporain, numéro dirigé par Bernadette Bost et Mireille LOSCO-LENA, «Recherches \& Travaux», n. 69, 2007, pp. 130.

1 Il volume si dispone in tre parti: «Autour de Serge Valletti» (pp. 7-36), «Sur quelques dramaturgies européennes» (pp. 37-108), «Paroles d'auteurs» (pp. 109-125). I contributi presentano un nuovo momento di attività critica, che approfondisce punti centrali del comico nei testi teatrali di Valletti, di Copi e di Novarina, in una prospettiva d'assieme rivolta anche al teatro europeo non di lingua francese degli ultimi decenni, per includervi infine riflessioni e commenti di autori viventi, a testimoniare le difficoltà del mestiere e integrare la varietà dell'esposizione.

2 In Serge Valletti ou les jeux de l'abondance digressive (pp. 9-15), chiarita la dominanza verbale dei numerosi e articolati testi del «mythomane professionnel», Jean-Pierre RYNGAERT propone di studiarne il discorso attraverso la formulazione di otto «Lois de l'abondance digressive», una serie di osservazioni volte a riconoscere dei «principes récurrents» persino nel «discours en apparence le plus désorganisé».

Mireille Losco-LENA in Serge Valletti: portrait de l'artiste en Don Quichotte (pp. 17-25) individua nell'eroe di Cervantes «la figure tutélaire de tous les personnages mythomanes de Valletti»: il desiderio di aventure, l'idea di hasard e la jubilation sono $\mathrm{i}$ principali elementi di un meccanismo comico che argomenta le «impressions» di un mondo reale poi trasposto sulla scena in una dimensione di «folie baroque». 
Più mirata all'analisi di singole rappresentazioni è la strategia di Bernadette BosT, De la tribu Léopold à la bande à Branko. Introduction à l'étude de l'homo valletticus (pp. 27-36). Il personaggio è visto secondo tre problematiche: la «découverte de son langage» (senso dell'ellissi, fantasie sintattiche, aforismi singolari), «l'individu et le groupe, structures socio-économiques» (habitat, nuclei familiari, differenze sociali, blande preoccupazioni di ordine morale), e «culture communautaire et transcendance...» (socialità rafforzata dal vino, dai giochi e dalle feste, con tracce di ritualità).

5 La valenza sovversiva della parodia nell'usurpare la performatività programmata di precisi elementi della messinscena è costitutiva del Rire comme une folle... Sur d'éventuels effets politiques de la parodie. "La Tour de la Défense" de Copi (pp. 77-89) di Olivier NEVEUX. Si tratta di aprirsi alla possibilità «d'un théâtre qui emprunterait ses codes à l'idéologie dominante et l'entraînerait en un endroit que celui-ci ne peut contenir». Il boulevard è in Copi il luogo di questa opposizione: un boulevard gay e lesbico dove si rivela uno humour camp, modelli comunicativi lontani da quelli della tradizionale forma di teatro 'popolare'. La proposta di 'lettura' considera la pièce un testo di «résistance [...] pour mettre en crise le caractère joué, fabriqué en toutes circonstances de l'ordre social et sexuel».

Didier PLASSARD, L'acrobate et le clown: notes sur la corporéité comique dans l'œuvre de Valère Novarina (pp. 91-95), traccia la cronologia di testi che portano l'eco del comico del circo: clown, artisti di music-hall, attori comici, l'acrobata che cade sono modelli di un universo funzionalizzato all'«étrangeté, tout à la fois comique et terrifiante, du lien du corps et de la parole».

7 Problemi peculiari del comico del discorso in Thierry toulze, Les 400 coups d'un Écolier Sacripant... (pp. 97-108). Riconosciuti nel personaggio alcuni tratti di Novarina, si constata che essi sembrano procedere «moins d'un désir violent d'anarchie que d'une incapacité fondamentale à accepter la réalité et à produire du sens à partir du langage».

8 Il diverso combinarsi delle analisi, convergenti nel focalizzare il punto di vista sui poteri del linguaggio, ha il merito di muovere dall'interpretazione di testi isolati per aggiungere nuovi elementi alla strategia complessiva del comico. 